\title{
Non-lattice simulation for supersymmetric gauge theories in one dimension
}

\author{
Masanori Hanada $^{1,2}$ * Jun Nishimura ${ }^{3,4}$ 团 and Shingo Takeuchi团 \\ ${ }^{1}$ Department of Physics, Kyoto University, Kitashirakawa, Kyoto 606-8502, Japan \\ ${ }^{2}$ Theoretical Physics Laboratory, RIKEN Nishina Center, 2-1 Hirosawa, Wako, Saitama 351-0198, Japan \\ ${ }^{3}$ High Energy Accelerator Research Organization (KEK), Tsukuba 305-0801, Japan \\ ${ }^{4}$ Department of Particle and Nuclear Physics, School of High Energy Accelerator Science, \\ Graduate University for Advanced Studies (SOKENDAI), Tsukuba 305-0801, Japan
}

(Dated: June, 2007; preprint: RIKEN-TH-104, KEK-TH-1158)

\begin{abstract}
Lattice simulation of supersymmetric gauge theories is not straightforward. In some cases the lack of manifest supersymmetry just necessitates cumbersome fine-tuning, but in the worse cases the chiral and/or Majorana nature of fermions makes it difficult to even formulate an appropriate lattice theory. We propose to circumvent all these problems inherent in the lattice approach by adopting a non-lattice approach in the case of one-dimensional supersymmetric gauge theories, which are important in the string/M theory context.
\end{abstract}

PACS numbers: 11.10.Kk; 11.15.Ha; 11.30.Pb

Introduction. - Lattice gauge theory, together with the developments of various simulation techniques, has provided us with a powerful non-perturbative method to study gauge theories such as QCD. However, when one tries to apply the method to supersymmetric gauge theories, which are interesting for many reasons, one has to face with some practical and theoretical obstacles.

First of all, since the algebra of supersymmetry contains continuous translations, which are broken to discrete ones, it seems unavoidable to break it on the lattice. Then, one has to include all the relevant terms allowed by symmetries preserved on the lattice, and fine-tune the coupling constants to arrive at the desired supersymmetric fixed point in the continuum limit. Recent progress (See ref. [1] and references therein.) is that one can reduce the number of parameters to be fine-tuned (even to zero in some cases) by preserving some part of supersymmetry. In lower dimensions, one can alternatively take the advantage of super-renormalizability, and determine the appropriate counter-terms by perturbative calculations to avoid fine-tuning. These two approaches can be nicely illustrated in one dimension by the example of a supersymmetric anharmonic oscillator [2, 3].

In the string/M theory context, supersymmetric gauge theories appear in various ways. In particular, the lowenergy effective theory of a stack of $N p$-branes are given by $(p+1)$-dimensional $\mathrm{U}(N)$ super Yang-Mills theory. This led to various interesting conjectures. For instance, the gauge/gravity duality states that the strong coupling limit of large- $N$ gauge theories has a dual description in terms of classical supergravity. A different but closely related conjecture asserts that non-perturbative formulations of superstring/ $\mathrm{M}$ theory can be given by matrix models, which can be obtained by dimensionally reducing 10d $\mathcal{N}=1 \mathrm{U}(N)$ super Yang-Mills theory to $D=0,1,2$ dimensions. In particular, the $D=1$ model [4] corresponds to the M Theory. Its bosonic version has been studied by Monte Carlo simulation using the lattice for- mulation [5] and the continuum quenched Eguchi-Kawai model [6]. However, the next step of including fermionic matrices would be difficult even theoretically because of their Majorana-Weyl nature inherited from 10d. See ref. 7] for a proposal using lattice, which preserves half of SUSY at the expense of breaking the $\mathrm{SO}(9)$ symmetry.

The aim of this letter is to point out that there exists an extremely simple and elegant method to simulate supersymmetric gauge theories in one dimension. (We discuss the case of $\mathrm{U}(N)$ gauge group, but extension to more general group must be possible.) In particular, it will enable us to study the gauge theory side of the aforementioned duality and also to put M theory on computer.

Let us first recall that the importance of the lattice formulation lies in its manifest gauge invariance. In the present $1 \mathrm{~d}$ case, however, the gauge dynamics is almost trivial. (We assume that the $1 \mathrm{~d}$ direction is compact. The non-compact case would be easier since the gauge dynamics is completely trivial.) This gives us an opportunity to use a non-lattice formulation. More specifically, we first take the static diagonal gauge. Using the residual large gauge transformation, we can choose a gauge slice such that the diagonal elements of the constant gauge field lie within a minimum interval. Finally we expand the fields into Fourier modes, and keep only the modes up to some cutoff. The crucial point of our method is that the gauge symmetry is completely fixed (up to the global permutation group, which is kept intact) before introducing the cutoff. This is specific to one dimension, and the momentum cutoff regularization in higher dimensions generally breaks gauge invariance.

Supersymmetric anharmonic oscillator. - To gain some insight into our new approach, we first apply it to a non-gauge supersymmetric theory, which is well studied by the lattice formulation. In particular, supersymmetry, which is broken by the cutoff in our formalism, is restored much faster than the continuum limit is achieved.

While the manuscript was being prepared, we received 
a preprint [8], in which the same model is studied on the lattice using various methods. As far as non-gauge theories are concerned, our approach is almost equivalent to the method with the non-local SLAC derivative [9]. The only difference is the identification of the modes at the boundary of the Brillouin zone in the lattice case. As a consequence, our results shown in fig. 1 agree with the corresponding results in ref. [8].

The model is defined by the action

$$
S=\int_{0}^{\beta} d t\left[\frac{1}{2}\left\{\left(\partial_{t} \phi\right)^{2}+h^{\prime}(\phi)^{2}\right\}+\bar{\psi}\left(\partial_{t}+h^{\prime \prime}(\phi)\right) \psi\right]
$$

where $\phi$ is a real scalar field, and $\psi$ is a one-component Dirac field, both in 1d, obeying periodic boundary conditions. This model is supersymmetric for arbitrary function $h(\phi)$, but here we take $h(\phi)=\frac{1}{2} m \phi^{2}+\frac{1}{4} g \phi^{4}$. In our approach we make a Fourier expansion

$$
\phi(t)=\sum_{n=-\Lambda}^{\Lambda} \tilde{\phi}_{n} \mathrm{e}^{i \omega n t} ; \quad \omega \equiv \frac{2 \pi}{\beta}
$$

and similarly for the fermionic fields, where $n$ takes integer values, and $\Lambda$ is the UV cutoff. In terms of the Fourier modes, the action can be written as $S=S_{\mathrm{B}}+S_{\mathrm{F}}$, where

$$
\begin{aligned}
S_{\mathrm{B}}= & \beta\left[\sum_{n=-\Lambda}^{\Lambda} \frac{1}{2}\left\{(n \omega)^{2}+m^{2}\right\} \tilde{\phi}_{n} \tilde{\phi}_{-n}\right. \\
& \left.+m g\left(\tilde{\phi}^{4}\right)_{0}+\frac{1}{2} g^{2}\left(\tilde{\phi}^{6}\right)_{0}\right] \\
S_{\mathrm{F}}= & \sum_{n k} \tilde{\bar{\psi}}_{n} \mathcal{M}_{n k} \tilde{\psi}_{k} \\
\mathcal{M}_{n k}= & \beta\left[(i n \omega+m) \delta_{n k}+3 g\left(\tilde{\phi}^{2}\right)_{l} \delta_{n, k+l}\right] .
\end{aligned}
$$

We have introduced a shorthand notation

$$
\left(f^{(1)} \cdots f^{(p)}\right)_{n} \equiv \sum_{k_{1}+\cdots+k_{p}=n} f_{k_{1}}^{(1)} \cdots f_{k_{p}}^{(p)} .
$$

Integrating out the fermions first, we obtain the effective action for the bosons as

$$
S_{\text {eff }}=S_{\mathrm{B}}-\ln \operatorname{det} \mathcal{M}
$$

where the fermion determinant $\operatorname{det} \mathcal{M}$ is real positive for positive $m$ and $g$.

As an efficient algorithm to simulate the model (6), we use the idea of the hybrid Monte Carlo algorithm [10]. Let us introduce the auxiliary real field $\Pi(t)$, whose Fourier components are denoted as $\tilde{\Pi}_{n}$, and consider the action

$$
S_{\mathrm{HMC}}=S_{\mathrm{eff}}+\sum_{n=-\Lambda}^{\Lambda} \frac{1}{2} \tilde{\Pi}_{n} \tilde{\Pi}_{-n}
$$

Integrating out the auxiliary field first, we retrieve (6). In order to update the fields, we solve the equations

$$
\begin{aligned}
\frac{d \tilde{\phi}_{n}(\tau)}{d \tau} & =\alpha_{n} \frac{\partial S_{\mathrm{HMC}}}{\partial \tilde{\Pi}_{n}}=\alpha_{n} \tilde{\Pi}_{-n} \\
\frac{d \tilde{\Pi}_{n}(\tau)}{d \tau} & =-\alpha_{n} \frac{\partial S_{\mathrm{HMC}}}{\partial \tilde{\phi}_{n}}=-\alpha_{n} \frac{\partial S_{\mathrm{eff}}}{\partial \tilde{\phi}_{n}}
\end{aligned}
$$

along the fictitious time $\tau$ for a fixed interval $\tau_{\mathrm{f}}$. The real coefficients $\alpha_{n}$ should be optimized based on the idea of the Fourier acceleration [11]. This evolution, if treated exactly, conserves the action $S_{\mathrm{HMC}}$. In practice, we discretize the $\tau$-evolution in such a way (the leap-frog discretization) that the reversibility is maintained. Due to the discretization, the action $S_{\mathrm{HMC}}$ changes by a small amount, say $\Delta S_{\mathrm{HMC}}$. In order to satisfy the detailed balance, we accept the new configuration with the probability $\min \left(1, \mathrm{e}^{-\Delta S_{\mathrm{HMC}}}\right)$, which is the usual Metropolis procedure. Before we start a new $\tau$-evolution, we refresh the $\tilde{\Pi}_{n}$ variables by drawing Gaussian random numbers which follow from the action (7). This procedure is necessary for avoiding the ergodicity problem. (The step size $\Delta \tau$ should be optimized for fixed $\tau_{\mathrm{f}}$ by maximizing the acceptance rate times $\Delta \tau$. Then $\tau_{\mathrm{f}}$ should be optimized by minimizing the autocorrelation time in units of step in the $\tau$-evolution.)

The main part of the computation is the evaluation of the term in eq. (9) given by

$$
\begin{aligned}
\frac{\partial S_{\text {eff }}}{\partial \tilde{\phi}_{n}}= & \beta\left[\left\{(n \omega)^{2}+m^{2}\right\} \tilde{\phi}_{-n}+4 m g\left(\tilde{\phi}^{3}\right)_{-n}\right. \\
& \left.+3 g^{2}\left(\tilde{\phi}^{5}\right)_{-n}\right]-\operatorname{tr}\left(\frac{\partial \mathcal{M}}{\partial \tilde{\phi}_{n}} \mathcal{M}^{-1}\right) .
\end{aligned}
$$

The convolution requires $\mathrm{O}\left(\Lambda^{2}\right)$ calculations, while the inverse $\mathcal{M}^{-1}$ requires $\mathrm{O}\left(\Lambda^{3}\right)$ calculations.

As usual, we extract masses from the exponential decay of the two-point functions

$$
\begin{aligned}
& G_{\mathrm{B}}(t) \equiv\langle\phi(0) \phi(t)\rangle=b_{0}+2 \sum_{n=1}^{\Lambda} b_{n} \cos (\omega n t), \\
& G_{\mathrm{F}}(t) \equiv\langle\psi(0) \bar{\psi}(t)\rangle=\sum_{n=-\Lambda}^{\Lambda} c_{n} \mathrm{e}^{-i \omega n t},
\end{aligned}
$$

where we have defined $b_{n} \equiv\left\langle\left|\tilde{\phi}_{n}\right|^{2}\right\rangle$ and $c_{n} \equiv\left\langle\left(\mathcal{M}^{-1}\right)_{n n}\right\rangle$. For the fermion, it proved convenient to consider, instead of (13), a symmetrized correlator

$$
\begin{aligned}
G_{\mathrm{F}}^{(\mathrm{sym})}(t) & \equiv \frac{1}{2}\left\{G_{\mathrm{F}}(t)+G_{\mathrm{F}}(-t)\right\} \\
& =c_{0}+2 \sum_{n=1}^{\Lambda} \operatorname{Re}\left(c_{n}\right) \cos (\omega n t),
\end{aligned}
$$

where we have used the fact $\left(\mathcal{M}_{n k}\right)^{*}=\mathcal{M}_{-n,-k}$. In fact the functions (12) and (14) with respect to $t$ oscillate with 
the frequency of the order of cutoff. This is nothing but the Gibbs phenomenon due to the sharp cutoff in the sum over Fourier modes. To overcome this problem, we note that the coefficients $b_{n}$ behave as $b_{n} \sim \frac{d_{1}}{n^{2}}+\frac{d_{2}}{n^{4}}$ at large $n$ as can be shown from general arguments. We obtain the coefficients $d_{1}$ and $d_{2}$ from the results at $n=\Lambda-1, \Lambda$, and extend the sum in (12) over $n$ up to 1000 assuming the above asymptotic form. We make an analogous analysis for $\operatorname{Re}\left(c_{n}\right)$ in (14). In this way we are able to see clear exponential behaviors, and extract the corresponding masses. The results for $\beta=1, m=10, g=100$ are plotted against $1 / \Lambda$ in fig. 1 (Note that the effective coupling constant is $g / m^{2}=1$.) We find that the finite $\Lambda$ effects are $\mathrm{O}(1 / \Lambda)$, and that the data points for the boson and the fermion lie on top of each other. Thus in our formalism, the effect of supersymmetry breaking by the cutoff disappears much faster than $1 / \Lambda$. In the same figure we also plot the results obtained from lattice formulations for comparison. (Matching the number of degrees of freedom, we make an identification $\Lambda=\frac{L}{2}$, where $L$ is the number of sites.)

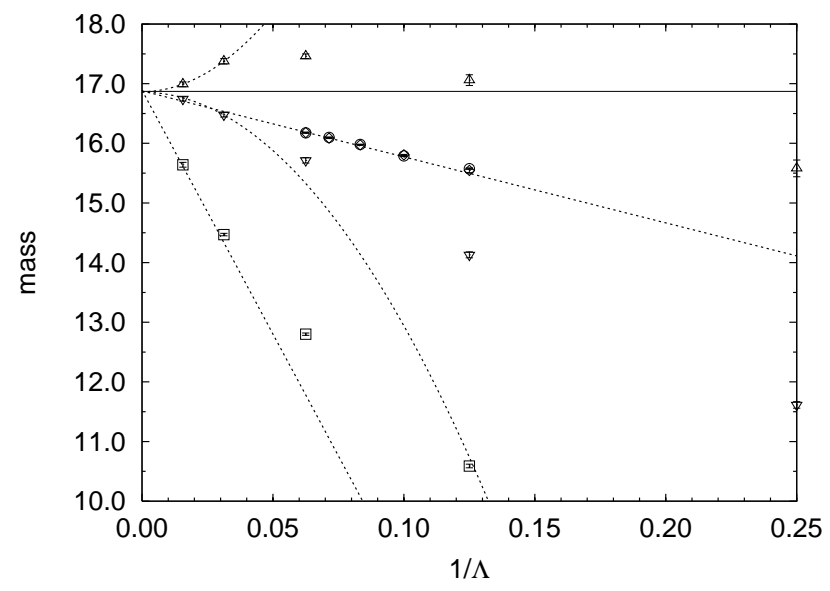

FIG. 1: The circles (diamonds) are the mass for the boson (fermion) obtained by our method for $\Lambda=8,10,12,14,16$. The triangles (inverted triangles) are the mass for the boson (fermion) obtained by Giedt et al. [1] with the $\mathrm{O}(a)$-improved lattice action, and the squares are the results obtained by Catterall and Gregory [2] with the lattice action preserving half of SUSY, hence degenerate. The horizontal line represents the exact result, and the dotted lines represent the expected behaviors at large $\Lambda$.

Supersymmetric matrix quantum mechanics. - Here we consider a model with four supercharges defined by

$$
\begin{aligned}
S= & \frac{1}{g^{2}} \int_{0}^{\beta} d t \operatorname{tr}\left\{\frac{1}{2}\left(D_{t} X_{i}\right)^{2}-\frac{1}{4}\left[X_{i}, X_{j}\right]^{2}\right. \\
& \left.+\bar{\psi} D_{t} \psi-\bar{\psi} \sigma_{i}\left[X_{i}, \psi\right]\right\},
\end{aligned}
$$

where $D_{t}=\partial_{t}-i[A(t), \cdot]$ represents the covariant derivative with the gauge field $A(t)$ being an $N \times N$ Hermitian matrix. The bosonic matrices $X_{i}(t)(i=1,2,3)$ are $N \times N$ Hermitian, and the fermionic matrices $\psi_{\alpha}(t)$ and $\bar{\psi}_{\alpha}(t)(\alpha=1,2)$ are $N \times N$ matrices with complex Grassmann entries. The $2 \times 2$ matrices $\sigma_{i}$ are the Pauli matrices. The model can be obtained formally by dimensionally reducing $4 \mathrm{~d} \mathcal{N}=1 \mathrm{U}(N)$ super Yang-Mills theory to $1 \mathrm{~d}$, and it can be viewed as a one-dimensional $\mathrm{U}(N)$ gauge theory. (The totally reduced model has been studied by Monte Carlo simulation in refs. [12].) Let us assume the boundary conditions to be periodic for bosons and anti-periodic for fermions. The extent $\beta$ in the Euclidean time direction then corresponds to the inverse temperature $\beta \equiv 1 / T$. The parameter $g$ in (15) can always be absorbed by an appropriate rescaling of the matrices and the time coordinate $t$. Hence we set $g=\frac{1}{\sqrt{N}}$ without loss of generality.

Let us take the static diagonal gauge $A(t)=$ $\frac{1}{\beta} \operatorname{diag}\left(\alpha_{1}, \cdots, \alpha_{N}\right)$, where $\alpha_{a}(a=1, \cdots, N)$ can be chosen to lie within the interval $(-\pi, \pi]$ by making a gauge transformation with a non-zero winding number [13]. We have to add to the action a term

$$
S_{\mathrm{FP}}=-\sum_{a<b} 2 \ln \left|\sin \frac{\alpha_{a}-\alpha_{b}}{2}\right|,
$$

which appears from the Faddeev-Popov procedure, and the integration measure for $\alpha_{a}$ is taken to be uniform.

We make a Fourier expansion

$$
X_{i}^{a b}(t)=\sum_{n=-\Lambda}^{\Lambda} \tilde{X}_{i n}^{a b} \mathrm{e}^{i \omega n t} ; \psi_{\alpha}^{a b}(t)=\sum_{r=-\lambda}^{\lambda} \tilde{\psi}_{\alpha r}^{a b} \mathrm{e}^{i \omega r t}
$$

and similarly for $\bar{\psi}$, where $r$ takes half-integer values, due to the anti-periodic boundary conditions, and $\lambda \equiv$ $\Lambda-1 / 2$. Eq. (15) can then be written as

$$
\begin{aligned}
S= & N \beta\left[\frac{1}{2} \sum_{n=-\Lambda}^{\Lambda}\left\{n \omega-\frac{\alpha_{a}-\alpha_{b}}{\beta}\right\}^{2} \tilde{X}_{i,-n}^{b a} \tilde{X}_{i n}^{a b}\right. \\
& \left.-\frac{1}{4} \operatorname{tr}\left(\left[\tilde{X}_{i}, \tilde{X}_{j}\right]^{2}\right)_{0}\right] \\
& +N \beta \sum_{r=-\lambda}^{\lambda}\left[i\left\{r \omega-\frac{\alpha_{a}-\alpha_{b}}{\beta}\right\} \tilde{\bar{\psi}}_{\alpha r}^{b a} \tilde{\psi}_{\alpha r}^{a b}\right. \\
& \left.-\left(\sigma_{i}\right)_{\alpha \beta} \operatorname{tr}\left\{\tilde{\bar{\psi}}_{\alpha r}\left(\left[\tilde{X}_{i}, \tilde{\psi}_{\beta}\right]\right)_{r}\right\}\right] .
\end{aligned}
$$

The algorithm for simulating (18) is analogous to the previous model. Here we introduce the auxiliary variables $\Pi_{i}(t)$ and $p_{a}$, which are $N \times N$ Hermitian matrices conjugate to $X_{i}(t)$ and $N$ real variables conjugate to $\alpha_{a}$, respectively. The fermion determinant is real positive, and the computational effort for one step in the $\tau$-evolution is proportional to $\Lambda^{3} N^{6}$. Figures 2 and 3 show the results for the energy and the Polyakov line, respectively, for the bosonic and supersymmetric cases. 


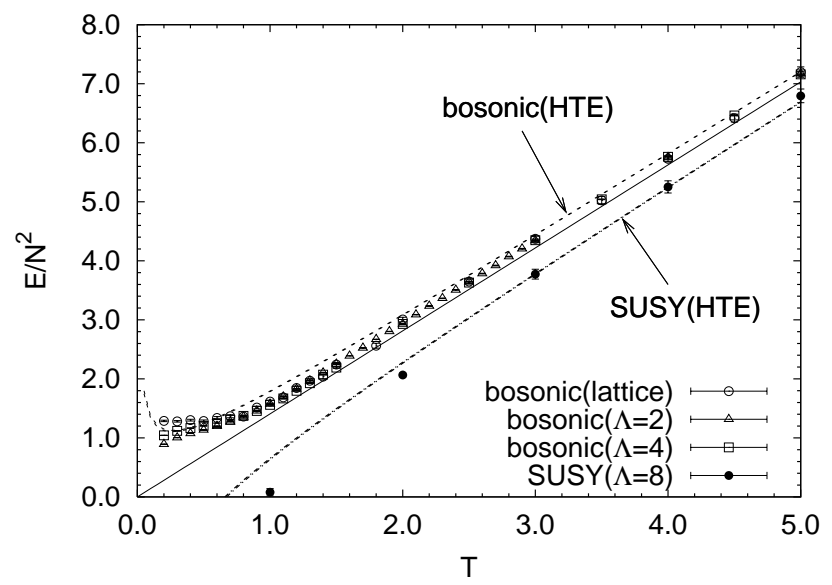

FIG. 2: The energy (normalized by $N^{2}$ ) is plotted against temperature for the matrix quantum mechanics with $N=4$.

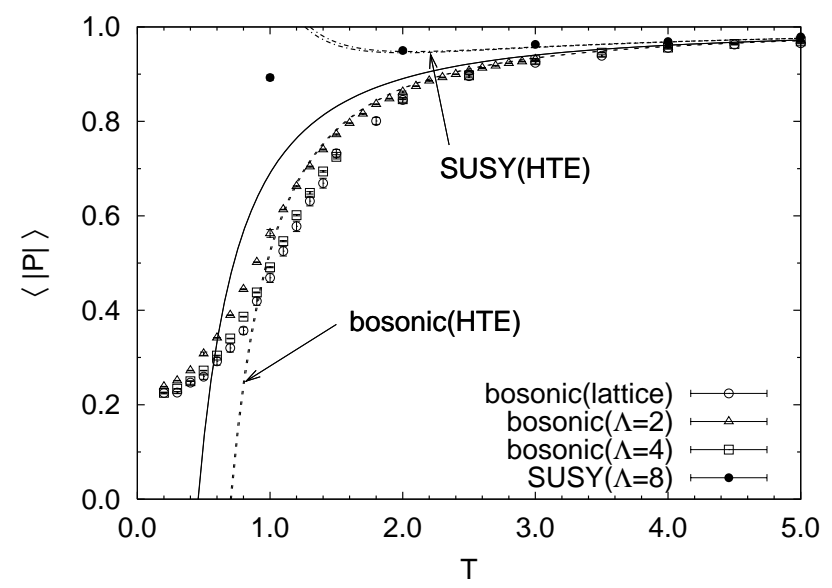

FIG. 3: The same as fig. 2 but for the absolute value of the Polyakov line.

In the bosonic case we also plot the results from lattice simulation with the lattice spacing $a=0.02$. (The number of lattice sites is given by $L=1 /(T a)$, which is 50 for $T=1$.) The results obtained by our new method approach the lattice result as $\Lambda$ is increased.

In the supersymmetric case, our preliminary results with $\Lambda=8$ reproduce the asymptotic behavior at large $T$ obtained by the high temperature expansion (HTE) [14] up to the next-leading order. (The solid lines represent the results at the leading order of HTE, which are the same for the bosonic and SUSY cases.) Note that our method is applicable also at low temperature, where the HTE is no more valid.

Summary and concluding remarks. - In this letter we have proposed a new simulation method, which enables non-perturbative studies of supersymmetric gauge theories in one dimension. For practical implementation, the idea of the hybrid Monte Carlo algorithm seems to be quite useful. In particular, the Fourier acceleration requires no extra cost, since we deal with the Fourier modes directly. The continuum limit is achieved much faster than one would expect naively from the number of degrees of freedom. This is understandable since the Fourier modes omitted by the cutoff scheme are naturally suppressed by the kinetic term.

It is straightforward to apply our method to the most interesting case with sixteen supercharges, which is currently under investigation [15].

Acknowledgements. - The authors would like to thank S. Catterall, Y. Kikukawa and F. Sugino for valuable discussions. The simulation has been performed using Yukawa Institute Computer Facility, RIKEN Super Combined Cluster System and a PC cluster at KEK.

* Electronic address: hana@riken.jp

† Electronic address: jnishi@post.kek.jp

‡ Electronic address: shingo@post.kek.jp

[1] J. Giedt, PoS LAT2006, 008 (2006).

[2] S. Catterall and E. Gregory, Phys. Lett. B 487, 349 (2000).

[3] J. Giedt, R. Koniuk, E. Poppitz and T. Yavin, JHEP 0412, 033 (2004).

[4] T. Banks, W. Fischler, S. H. Shenker and L. Susskind, Phys. Rev. D 55, 5112 (1997).

[5] R. A. Janik and J. Wosiek, Acta Phys. Polon. B 32, 2143 (2001); P. Bialas and J. Wosiek, Nucl. Phys. Proc. Suppl. 106, 968 (2002); O. Aharony, J. Marsano, S. Minwalla and T. Wiseman, Class. Quant. Grav. 21, 5169 (2004); N. Kawahara, J. Nishimura and S. Takeuchi, JHEP 0705, 091 (2007).

[6] N. Kawahara and J. Nishimura, JHEP 0509, 040 (2005).

[7] D. B. Kaplan and M. Unsal, JHEP 0509, 042 (2005).

[8] G. Bergner, T. Kaestner, S. Uhlmann and A. Wipf, arXiv:0705.2212 [hep-lat].

[9] S. D. Drell, M. Weinstein and S. Yankielowicz, Phys. Rev. D 14, 487 (1976); Phys. Rev. D 14, 1627 (1976).

[10] S. Duane, A. D. Kennedy, B. J. Pendleton and D. Roweth, Phys. Lett. B 195, 216 (1987).

[11] S. Catterall and S. Karamov, Phys. Lett. B 528, 301 (2002).

[12] W. Krauth, H. Nicolai and M. Staudacher, Phys. Lett. B 431, 31 (1998); J. Ambjorn, K. N. Anagnostopoulos, W. Bietenholz, T. Hotta and J. Nishimura, JHEP 0007, 013 (2000); J. Ambjorn, K. N. Anagnostopoulos, W. Bietenholz, F. Hofheinz and J. Nishimura, Phys. Rev. D 65, 086001 (2002); Z. Burda, B. Petersson and M. Wattenberg, JHEP 0503, 058 (2005).

[13] In the present case with only adjoint representations, it is more convenient to impose a weaker constraint $\max _{i}\left(\alpha_{i}\right)-\min _{i}\left(\alpha_{i}\right)<2 \pi$, which respects the center $\mathrm{U}(1)$ symmetry $\alpha_{i} \mapsto \alpha_{i}+$ const..

[14] N. Kawahara, J. Nishimura and S. Takeuchi, in preparation.

[15] K. N. Anagnostopoulos, M. Hanada, J. Nishimura and S. Takeuchi, work in progress. 